\title{
Effect of the Structure of Seven Anthocyanins on Self-association and Colour in an Aqueous Alcohol Solution
}

Fu Liang $\operatorname{Han}^{1 *}$, Yan $\mathrm{Xu}^{2}$

(1) College of Enology, Northwest A\&F University, Yangling, Shaanxi, 712100, China

(2) Centre for Brewing Science and Enzyme Biotechnology, School of Biotechnology, Jiangnan University, Wuxi, Jiangsu, 214122, China

Submitted for publication: June 2014

Accepted for publication: August 2014

Key words: wine, anthocyanin structure, self-association, colour

\begin{abstract}
Anthocyanins play an important role in the colour of red wine or mulberry wine. The effect of anthocyanin structures (substitution therein) on the self-association and colour in an aqueous alcohol solution was investigated in order to provide helpful knowledge about the relationship between anthocyanin structure and colour. The addition of hydroxyl at the $\mathrm{C} 3$ ' position weakens self-association, whereas addition at the C5' position facilitates self-association. However, the hydroxyl substitutions at the C3' and C5' positions enhance colour. The methoxy substitutions at the $\mathrm{C} 3$ ' and $\mathrm{C5}^{\prime}$ positions enhance the self-association and the colour. The replacement of glucoside with the disaccharide rutinoside at the $\mathbf{C} 3$ position was detrimental to self-association, but the substitution of rutinoside at the $\mathrm{C} 3$ position produced a stronger colour than the substitution of glucoside at the same position. Cyanidin 3-O-glucoside and cyanidin 3-O-rutinoside enhanced colour via intramolecular co-pigmentation instead of self-association. The anthocyanin colour was affected synthetically by the types and positions of the substituent in the anthocyanin molecule.
\end{abstract}

\section{INTRODUCTION}

Colour is an important organoleptic property of red wine. Anthocyanins give rise to a wide range of colours, from orange to blue, in red wine (Waterhouse \& Kennedy, 2004; Oliveira et al., 2006; 2010). Anthocyanins in grapes and wines are based on five or six aglycones (pelargonidin, cyanidin, delphinidin, peonidin, petunidin and malvidin), with different patterns of substitution in the B ring (Fig. 1). They exist exclusively as 3-glucosides in Vitis vinifera varieties, while 3,5-diglucosides exist in Chinese species (Vitis amurensis), American species (V. riparia, V. rupestris, etc.), and the hybrid varieties (Alcalde-Eon et al., 2006; Liang et al., 2008; Han et al., 2009; He et al., 2010; Li et al., 2010; 2011). Cyanidin 3-O-glucoside and cyanidin 3-O-rutinoside are dominant in mulberry and its wine, while only trace amounts of pelargonidin 3-O-glucoside can be found (Fig. 1) (Dugo et al., 2001; Qin et al., 2010).

Co-pigmentation has an enhanced effect on the colour of red wine (Escribano-Bailón \& Santos-Buelga, 2012). It occurs via three types of molecular interactions. One is intermolecular co-pigmentation, which occurs between anthocyanins and other non-coloured or coloured copigments such as flavonoids, amino acids, organic acids and polysaccharides (Boulton, 2001; Eiro \& Heinonen, 2002; Castaneda-Ovando et al., 2009). Copigment molecules enhance the colour by impacting/stacking on the planar moieties of anthocyanin. Intramolecular co-pigmentation within an anthocyanin molecule can also cause a colour change in wines. In this case, the acyl or glycosyl groups interact with the $\pi$ system of the planar pyrylium ring of anthocyanin (Escribano-Bailón \& Santos-Buelga, 2012). In particular, intermolecular interactions among anthocyanin molecules can lead to co-pigmentation, called "selfassociation". Anthocyanin molecules stack vertically in chiral (helical) aggregates, mostly in a left-handed manner, and intensify in colour (Hoshino et al., 1981a; Dangles et al., 1993; Gonzáez-Manzano et al., 2008; Escribano-Bailón \& Santos-Buelga, 2012; Gavara et al., 2013).

Anthocyanins in red wines can exist in several structural conformations, and these structures are present in an equilibrium: the blue quinoidal base (A), the red flavylium ion $\left(\mathrm{AH}^{+}\right)$, the colourless pseudobase or carbinol pseudobase (B), and the colourless chalcone form (C) with three transitions, viz. cis-chalcone $\left(\mathrm{C}_{\mathrm{C}}\right)$, trans-chalcone $\left(\mathrm{C}_{\mathrm{t}}\right)$ and ionised cis-chalcone $\left(\mathrm{C}_{\mathrm{C}-}\right)$ (Cheminat \& Brouillard, 1986; Março \& Scarminio, 2007). The self-association between the flavylium cation and its chalcone form of anthocyanins has been confirmed, which is helpful for wine colour stability and enhancement (Houbiers et al., 1998). Anthocyanins with different structures can change the predominance of a structural conformation. Thus, anthocyanins contribute to different wine colours.

Self-association has been confirmed by a positive deviation from Beer's law. Self-association occurs in 


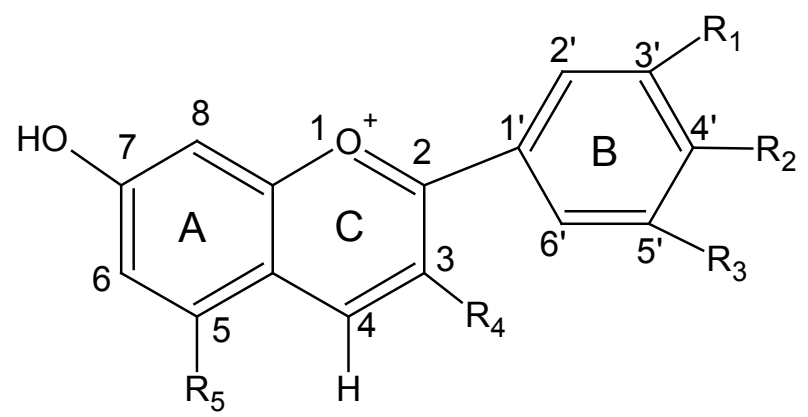

\begin{tabular}{llllll}
\hline Anthocyanins & $\mathrm{R}_{1}$ & $\mathrm{R}_{2}$ & $\mathrm{R}_{3}$ & $\mathrm{R}_{4}$ & $\mathrm{R}_{5}$ \\
\hline Pelargonidin 3-O-glucoside & $\mathrm{H}$ & $\mathrm{OH}$ & $\mathrm{H}$ & Glucoside & $\mathrm{OH}$ \\
Cyanidin 3-O-glucoside & $\mathrm{OH}$ & $\mathrm{OH}$ & $\mathrm{H}$ & Glucoside & $\mathrm{OH}$ \\
Delphinidin 3-O-glucoside & $\mathrm{OH}$ & $\mathrm{OH}$ & $\mathrm{OH}$ & Glucoside & $\mathrm{OH}$ \\
Peonidin 3-O-glucoside & $\mathrm{OCH}_{3}$ & $\mathrm{OH}$ & $\mathrm{H}$ & Glucoside & $\mathrm{OH}$ \\
Malvidin 3-O-glucoside & $\mathrm{OCH}_{3}$ & $\mathrm{OH}$ & $\mathrm{OCH}_{3}$ & Glucoside & $\mathrm{OH}$ \\
Malvidin 3,5-O-diglucoside & $\mathrm{OCH}_{3}$ & $\mathrm{OH}$ & $\mathrm{OCH}_{3}$ & Glucoside & Glucoside \\
Cyanidin 3-O-rutinoside & $\mathrm{OH}$ & $\mathrm{OH}$ & $\mathrm{H}$ & Rutinoside & $\mathrm{OH}$ \\
\hline
\end{tabular}

FIGURE 1

Structure of anthocyanins.

relatively concentrated solutions $(1 \mathrm{mM})$ of anthocyanins (Boulton, 2001; Cavalcanti et al., 2011). A previous study speculated that co-pigmentation, rather than self-association, was responsible for the enhanced but nonlinear colour contributions in red wine (Boulton, 2001). However, in winelike solution with anthocyanins in the concentration range of 50 to $600 \mathrm{mg} / \mathrm{L}$, the self-association was responsible for from $8 \%$ up to $60 \%$ of the increase in absorbance at $520 \mathrm{~nm}$ (Escribano-Bailón \& Santos-Buelga, 2012). It has been shown in a young red wine that self-association of malvidin 3-O-glucoside was more important than co-pigmentation (Lambert et al., 2011).

Several previous studies have proposed the effect of anthocyanin structure on colour (Hoshino et al., 1981a; Gonzáez-Manzano et al., 2008; Gavara et al., 2013). It has been reported previously that the presence of the methoxy group in the B-ring and of the glucose moiety at the C5 or C3 position of anthocyanins influences the self-association and colour appearance of anthocyanins. However, the effect of substituent groups of anthocyanins in wines on their selfassociation and colour remains unclear. In this study, the selfassociation and colour of seven anthocyanins in an aqueous alcohol solution ( $12 \%$ ethanol, $\mathrm{pH} 3.50)$ were investigated in order to provide helpful knowledge on the colour mechanism of anthocyanins in red wine or mulberry wine.

\section{MATERIALS AND METHODS}

\section{Reagents and chemicals}

Ethanol (99.9\%) and formic acid (98\%) were purchased from Sigma-Aldrich (Shanghai, China) and Anpel Company (Shanghai, China) respectively. Seven anthocyanins (pelargonidin 3-O-glucoside, cyanidin 3-O-glucoside, delphinidin 3-O-glucoside, peonidin 3-O-glucoside, malvidin 3-O-glucoside, malvidin 3,5-O-diglucoside and cyanidin 3-O-rutinoside) were purchased from Extrasynthese (Lyon, France).
Aqueous alcohol solution and solutions of anthocyanins The model wine solution was prepared by dissolving ethanol $(12 \% \mathrm{v} / \mathrm{v})$ in ultrapure water, and the $\mathrm{pH}$ of model wine solution was adjusted to 3.50 using formic acid $(2 \% \mathrm{v} / \mathrm{v})$.

The stock solution of each individual anthocyanin was prepared by dissolving commercial anthocyanins $(800.0$ $\mu \mathrm{mol} / \mathrm{L})$ in the hydroalcoholic solution containing $12 \%$ ethanol (10 mL, $\mathrm{pH} 3.50)$. The stock solution was diluted (64-, 32-, 16-, 8-, 4-, 2- and 0-fold) to generate a series of solutions with different anthocyanin concentrations $(\mathrm{L} 1=$ $12.5 \mu \mathrm{mol} / \mathrm{L}, \mathrm{L} 2=25.0 \mu \mathrm{mol} / \mathrm{L}, \mathrm{L} 3=50.0 \mu \mathrm{mol} / \mathrm{L}, \mathrm{L} 4=$ $100.0 \mu \mathrm{mol} / \mathrm{L}, \mathrm{L} 5=200.0 \mu \mathrm{mol} / \mathrm{L}, \mathrm{L} 6=400.0 \mu \mathrm{mol} / \mathrm{L}$ and $\mathrm{L} 7=800.0 \mu \mathrm{mol} / \mathrm{L})$. Each series of anthocyanin solution was prepared in duplicate.

\section{Estimation of self-association}

The method proposed by Boulton (1996) was used for estimating the self-association of anthocyanins in solution. The absorbance of anthocyanin solutions at $520 \mathrm{~nm}$ was measured using the UV/Vis spectrophotometer (Unico 2802 UV/Vis, Unico Instrument Co., Ltd., Shanghai, China). The absorbance value of the solution with the highest anthocyanin concentration, A, was recorded. After each dilution, the absorbance, Ad, and the dilution factor, $\mathrm{D}_{\mathrm{f}}$, were recorded. Self-association was then expressed as $\left(\mathrm{A}-\mathrm{A}_{\mathrm{d}} \times \mathrm{D}_{\mathrm{f}}\right) / \mathrm{A}_{\mathrm{d}} \times \mathrm{D}_{\mathrm{f}} \times 100$.

\section{Colour analyses using the CIELAB space}

Colour analyses using the CIELAB space were performed according to the published method (Ayala et al., 1997; 1999). Coordinate $a^{*}$ is related to red colour if $\mathrm{a}^{*}>0$ and to green colour if $\mathrm{a}^{*}<0$. Coordinate $\mathrm{b}^{*}$ is related to yellow colour if $b^{*}>0$ and to blue colour if $b^{*}<0$. L* (lightness) is the lightness of a coloured object judged relative to the lightness of what appears as white. The chroma is expressed as $\mathrm{C}=$ $\left(\mathrm{a}^{* 2}+\mathrm{b}^{* 2}\right)^{1 / 2}$, and the tone is expressed as $\mathrm{H}=\arctan \left(\mathrm{b}^{*} / \mathrm{a}^{*}\right)$. 
The colour difference is expressed as $\Delta \mathrm{E}=\left[(\Delta \mathrm{L})^{2}+\left(\Delta \mathrm{a}^{*}\right)^{2}+\right.$ $\left.\left(\Delta b^{*}\right)^{2}\right]^{1 / 2}$. The blank was the model wine solution.

\section{UV/Vis spectra}

A Unico $2802 \mathrm{UV} / \mathrm{Vis}$ spectrophotometer with a standard 96-well plate (quartz) was used to scan (200 to $700 \mathrm{~nm}$, path length $=2 \mathrm{~nm}$ ) the absorption spectra of seven anthocyanin solutions at their highest concentration.

\section{Purity analysis}

The purity of anthocyanins was assessed over a Kromasil $\mathrm{C}_{18}$ column $(250 \mathrm{~mm} \times 4.6 \mathrm{~mm}$, i.d., $5 \mu \mathrm{m})($ Eka Nobel $\mathrm{AB}$, Bohus, Sweden) using high performance liquid chromatography (HPLC, Dionex P680) at a flow rate of 1.0 $\mathrm{mL} / \mathrm{min}$ with a gradient of solvent $\mathrm{B}$ (acetonitrile/formic acid $(100 / 2, \mathrm{v} / \mathrm{v}))$ in $\mathrm{A}$ (water/formic acid $(100 / 2, \mathrm{v} / \mathrm{v}))$. A linear gradient was programmed as follows: 0 to $30 \mathrm{~min}$, 0 to $30 \% \mathrm{~B} ; 30$ to $35 \mathrm{~min}, 30$ to $100 \% \mathrm{~B} ; 35$ to $37 \mathrm{~min}$, 100 to $0 \% \mathrm{~B}$. The detection wavelength on the diode array detector was set at $520 \mathrm{~nm}$, the injection volume was 20 $\mu \mathrm{L}$, and the column temperature was maintained at $30^{\circ} \mathrm{C}$. The samples were analysed directly and peak areas were used to calculate the purity of the anthocyanins. The purity of pelargonidin 3-O-glucoside, cyanidin 3-O-glucoside, delphinidin 3-O-glucoside, peonidin 3-O-glucoside, malvidin 3-O-glucoside, malvidin 3,5-O-diglucoside, and cyaniding 3-O-rutinoside was $99.63 \%, 99.88 \%, 99.70 \%$, $99.40 \%, 99.16 \%, 96.46 \%$ and $99.61 \%$ respectively.

\section{RESULTS AND DISCUSSION}

The patterns of visible absorption spectra of these anthocyanins were dependent on the substitutions/structure of the B-ring of anthocyanins. The wavelength of maximum absorption of pelargonidin 3-O-glucoside, cyanidin 3-O-glucoside, delphinidin 3-O-glucoside, peonidin 3-O-glucoside and malvidin 3-O-glucoside shifted to higher wavelengths (bathochromic shift) with the increase in the number of hydroxy/methoxy groups in the B-ring (Table 1). These results are in accordance with a previous study (Cabrita et al., 2000). The addition of glucoside at position C5 in malvidin 3-O-glucoside or the substitution of glucoside with rutinoside at position $\mathrm{C} 3$ led to similar bathochromic shifts in the absorption maxima.

\section{Self-association of anthocyanins}

The value of self-association increased with an increase in dilution factor in the case of pelargonidin 3-O-glucoside and cyanidin 3-O-rutinoside (Table 2). In the case of the five other anthocyanins, self-association increased for dilution factors from 2 to 16 or 32 , and then declined at the highest dilution factors. This indicates that the dilution factor affected the value of self-association and the self-association was dependent on the concentration of anthocyanins.

Self-association of anthocyanins has been reported to occur in relatively concentrated solution $(1 \mathrm{mM})$ (Boulton, 2001). However, here it occurred in a concentrated solution of $0.8 \mathrm{mM}$. The self-association of anthocyanins was responsible for 15 to $60 \%$ of the absorbance at $520 \mathrm{~nm}$ across all dilutions; a similar result was observed in a previous study (Gonzáez-Manzano et al., 2008).
Pelargonidin 3-O-glucoside $(\mathrm{H}, \mathrm{OH}, \mathrm{H})$, cyanidin 3-Oglucoside $(\mathrm{OH}, \mathrm{OH}, \mathrm{H})$ and delphinidin 3-O-glucoside $(\mathrm{OH}$, $\mathrm{OH}, \mathrm{OH}$ ) differ in the number and sites of hydroxyl substitutions in the B-ring (Fig. 1). Comparing the self-association values in two- to 32-fold dilutions (Table 2), cyanidin 3-Oglucoside had the lowest value, followed by pelargonidin 3-O-glucoside, while delphinidin 3-O-glucoside showed the highest value. At 64-fold dilution, the self-association value of cyanidin $3-O$-glucoside was the smallest and that of pelargonidin 3-O-glucoside was the highest. These results indicate that the addition of hydroxyl at the C3' position weakened self-association, whereas addition at the $\mathrm{C}^{\prime}$ position facilitated self-association. In wine-like solutions, the various forms of anthocyanin structures exist in equilibrium (Cheminat \& Brouillard, 1986; Março \& Scarminio, 2007). The self-association of anthocyanin chromophores (flavylium or quinonoidal form) and their colourless forms (pseudobase or carbinol pseudobase, and chalcone form) has been reported, and the planar structure of anthocyanin aglycone favours self-association (Hoshino et al., 1981a; Houbiers et al., 1998). Self-association occurs by molecular stacking along either a left- or a right- handed helical axis (Hoshino et al., 1981a; 1981b; Ellestad, 2006). The non-planar structure between the C-ring and B-ring of anthocyanins can affect the chiral stacking (Sakata et al., 2006). Hydroxy substitution at the $\mathrm{C} 3$ ' position on the B-ring influenced the tauomeric distribution between anthocyanin chromophores and their colourless forms, and affected the non-planar structure between the C-ring and the B-ring. Thus, cyanidin 3-O-glucoside stacked along a different chiral (helical) axis than the other two anthocyanins, and showed different selfassociation values.

Pelargonidin 3-O-glucoside $(\mathrm{H}, \mathrm{OH}, \mathrm{H})$, peonidin 3-O-glucoside $\left(\mathrm{OCH}_{3}, \mathrm{OH}, \mathrm{H}\right)$ and malvidin 3-O-glucoside $\left(\mathrm{OCH}_{3}, \mathrm{OH}, \mathrm{OCH}_{3}\right)$ differ in their pattern of $-\mathrm{OCH}_{3}$ substitutions on the B-ring (Fig. 1). Among these three anthocyanins, malvidin 3-O-glucoside had the highest values of selfassociation at all dilutions, while pelargonidin 3-O-glucoside gave the smallest values. These results indicate that methoxy substitutions at the $\mathrm{C}^{\prime}$ ' and $\mathrm{C}^{\prime}$ ' positions on the B-ring enhance self-association via chiral stacking.

It is concluded that the magnitude of the self-association of these anthocyanins is determined by the degree of methoxylation on the B-ring (Gonzáez-Manzano et al., 2008). The results of this study are in agreement with the observation that the greater the degree of methoxylation of the B-ring in the anthocyanins, the greater the magnitude of

\section{TABLE 1}

Maximum absorption wavelength of the seven anthocyanins.

\begin{tabular}{ll}
\hline Anthocyanins & $\lambda_{\max }(\mathrm{nm})$ \\
\hline Pelargonidin 3-O-glucoside & 500 \\
Cyanidin 3- $O$-glucoside & 510 \\
Delphinidin 3- $O$-glucoside & 516 \\
Peonidin 3- $O$-glucoside & 512 \\
Malvidin 3- $O$-glucoside & 520 \\
Malvidin 3,5- $O$-diglucoside & 522 \\
Cyanidin 3- $O$-rutinoside & 514 \\
\hline
\end{tabular}


TABLE 2

The self-association of the seven anthocyanins.

\begin{tabular}{lllllll}
\hline Dilution factors & 2 & 4 & 8 & 16 & 32 & 64 \\
\hline Pelargonidin 3- $O$-glucoside & $31.5 \pm 1.18$ & $62.67 \pm 6.69$ & $81.79 \pm 7.71$ & $116.34 \pm 7.28$ & $106.82 \pm 13.29$ & $139.64 \pm 17.84$ \\
Cyanidin 3- $O$-glucoside & $30.64 \pm 1.17$ & $59.3 \pm 2.60$ & $71.23 \pm 6.00$ & $83.37 \pm 6.88$ & $88.3 \pm 4.84$ & $67.16 \pm 7.63$ \\
Delphinidin 3- $O$-glucoside & $40.41 \pm 0.56$ & $81.01 \pm 1.86$ & $105.81 \pm 7.22$ & $118.38 \pm 5.42$ & $126.32 \pm 5.82$ & $100.91 \pm 9.17$ \\
Peonidin 3-O-glucoside & $41.74 \pm 3.76$ & $89.51 \pm 5.76$ & $117.67 \pm 8.87$ & $145.43 \pm 19.28$ & $136.24 \pm 11.93$ & $107.25 \pm 18.32$ \\
Malvidin 3- $O$-glucoside & $65.03 \pm 9.05$ & $123.52 \pm 6.64$ & $162.18 \pm 10.44$ & $165.93 \pm 10.75$ & $165.93 \pm 10.75$ & $145.42 \pm 18.27$ \\
Malvidin 3,5-O-diglucoside & $53.22 \pm 4.86$ & $98.55 \pm 0.00$ & $125.3 \pm 16.77$ & $113.44 \pm 0.00$ & $140.12 \pm 37.73$ & $42.29 \pm 50.31$ \\
Cyanidin 3- $O$-rutinoside & $16.26 \pm 2.73$ & $36.93 \pm 0.99$ & $51.67 \pm 2.43$ & $60.76 \pm 1.82$ & $59.84 \pm 10.76$ & $68.2 \pm 15.86$ \\
\hline
\end{tabular}

Note: data are mean \pm standard deviation $(n=2)$

self-association in these three anthocyanins.

Pelargonidin 3-O-glucoside $(\mathrm{H}, \mathrm{OH}, \mathrm{H})$, cyanidin 3-O-glucoside $(\mathrm{OH}, \mathrm{OH}, \mathrm{H})$ and peonidin 3-O-glucoside $\left(\mathrm{OCH}_{3}, \mathrm{OH}, \mathrm{H}\right)$ differ in the substitutions at position $\mathrm{C}^{\prime}$ on the B-ring (Fig. 1). Across the two- to 32-fold dilutions, cyanidin 3-O-glucoside consistently had the smallest values of self-association, whereas peonidin 3-O-glucoside had the largest values. However, pelargonidin 3-O-glucoside displayed the largest value, while cyanidin 3-O-glucoside showed the smallest value at 64 -fold dilution. These results indicate that the addition of a methoxy group at the C3' position enhanced the self-association, but the addition of hydroxyl at the same position reduced the self-association.

Malvidin 3-O-glucoside and malvidin 3,5-O-diglucoside differ in the substitution of a glucoside at the C5' position (Fig. 1). Higher self-association was observed for malvidin 3-O-glucoside, which indicates that the addition of glucoside at the $\mathrm{C} 5$ position was detrimental to self-association. A previous report showed that malvidin 3-O-glucoside was less capable of self-aggregation than malvidin 3,5-O-diglucoside at pH 7.0 (Hoshino et al., 1981a). However, it should be noted that the current study was conducted at $\mathrm{pH} 3.50$ with the red flavylium ion $\left(\mathrm{AH}^{+}\right)$being the dominant form of anthocyanin. In addition, the glucoside at the $\mathrm{C}^{5}$ position of malvidin 3,5-O-diglucoside can sterically hinder its own chiral (helical) stacking (Hoshino et al., 1981b; EscribanoBailón \& Santos-Buelga, 2012).

Cyanidin 3-O-glucoside and cyanidin 3-O-rutinoside differ in the nature of the sugar at the $\mathrm{C} 3$ position (Fig. 1). Cyanidin 3-O-glucoside showed a higher level of self-association than cyanidin 3-O-rutinoside. This observation indicates that the replacement of glucoside with the disaccharide rutinoside at the $\mathrm{C} 3$ position hindered selfassociation.

\section{Effect of hydroxyl substitution on the B-ring on colour}

Pelargonidin 3-O-glucoside $(\mathrm{H}, \mathrm{OH}, \mathrm{H})$, cyanidin 3-O-glucoside $(\mathrm{OH}, \mathrm{OH}, \mathrm{H})$ and delphinidin 3-O-glucoside $(\mathrm{OH}$, $\mathrm{OH}, \mathrm{OH}$ ) differ in their pattern of $-\mathrm{OH}$ substitutions on the B-ring (Fig. 1). The wavelength of maximum absorption $\left(\lambda_{\max }\right)$ among these three compounds was the lowest for pelargonidin 3-O-glucoside and highest for delphinidin 3-Oglucoside, while cyanidin 3-O-glucoside had an intermediate value (Table 1). The difference between the values of $\lambda_{\text {max }}$ for pelargonidin 3-O-glucoside and cyanidin 3-O-glucoside was greater than that between cyanidin 3-O-glucoside and del- phinidin 3-O-glucoside (Table 1), which indicates that more hydroxyl substitutions on the B-ring caused a higher bathochromic shift, and hydroxyl substitution at the C3' position had a greater impact on the bathochromic shift than the same substitution at the C5' position.

Regarding the colour of these three anthocyanins (Fig. 2), it was found that the magnitude of the $\mathrm{L}$ value was pelargonidin 3-O-glucoside $>$ cyanidin 3-O-glucoside $>$ delphinidin 3-O-glucoside. Delphinidin 3-O-glucoside presented the highest $a^{*}$ values, followed by cyanidin 3-O-glucoside and pelargonidin 3-O-glucoside. Contrary to the $\mathrm{a}^{*}$ values, pelargonidin 3-O-glucoside showed the highest $\mathrm{b}^{*}$ value, followed by cyanidin $3-O$-glucoside and then delphinidin 3-O-glucoside. The magnitude of the difference of the $\mathrm{L}, \mathrm{a}^{*}, \mathrm{~b}^{*}$ values between pelargonidin 3-O-glucoside and cyanidin 3-O-glucoside or between cyanidin 3-O-glucoside and delphinidin 3-O-glucoside became larger with an increase in their concentrations. Their $\mathrm{C}$ values at concentrations L1 to L6 increased in the order of pelargonidin 3-O-glucoside, cyanidin 3-O-glucoside and delphinidin 3-O-glucoside. It decreased at level 7 in the order of pelargonidin 3-O-glucoside, cyanidin 3-O-glucoside and delphinidin 3-O-glucoside. The $\mathrm{H}$ values of the three anthocyanins decreased in the following order: pelargonidin 3-O-glucoside, cyanidin 3-O-glucoside and delphinidin 3-O-glucoside. The highest and the lowest $\Delta \mathrm{E}$ values were observed for pelargonidin 3-O-glucoside and delphinidin 3-O-glucoside respectively. The largest differences in $\Delta \mathrm{E}$ values were observed at level 7 . Their colour difference was visually discernible at levels 4 to 7 (Fig. 3), as the difference in the $\Delta \mathrm{E}$ value between pelargonidin 3-O-glucoside and cyanidin 3-O-glucoside, or between cyanidin 3-O-glucoside and delphinidin 3-O-glucoside was more than 1 . These results indicate that hydroxyl substitutions on the B-ring lead to the colour darkening ( $\mathrm{L}$ value decreases), increase the intensity of red ( $\mathrm{a}^{*}$ value increases) and blue colour ( $\mathrm{b}^{*}$ value decreases), reduce the intensity of yellow colour $(\mathrm{H}$ value decrease), and give rise to a purer and brighter chroma ( $\mathrm{C}$ value increases).

The magnitude of the difference in colour between pelargonidin 3-O-glucoside and cyanidin 3-O-glucoside was bigger than that between cyanidin 3-O-glucoside and delphinidin 3-O-glucoside (Fig. 2). This result coincided with the observation of their $\lambda_{\max }$. These results confirm that the higher number of hydroxyl substitutions on the B-ring led to large bathochromic shifts and changes in colour. 

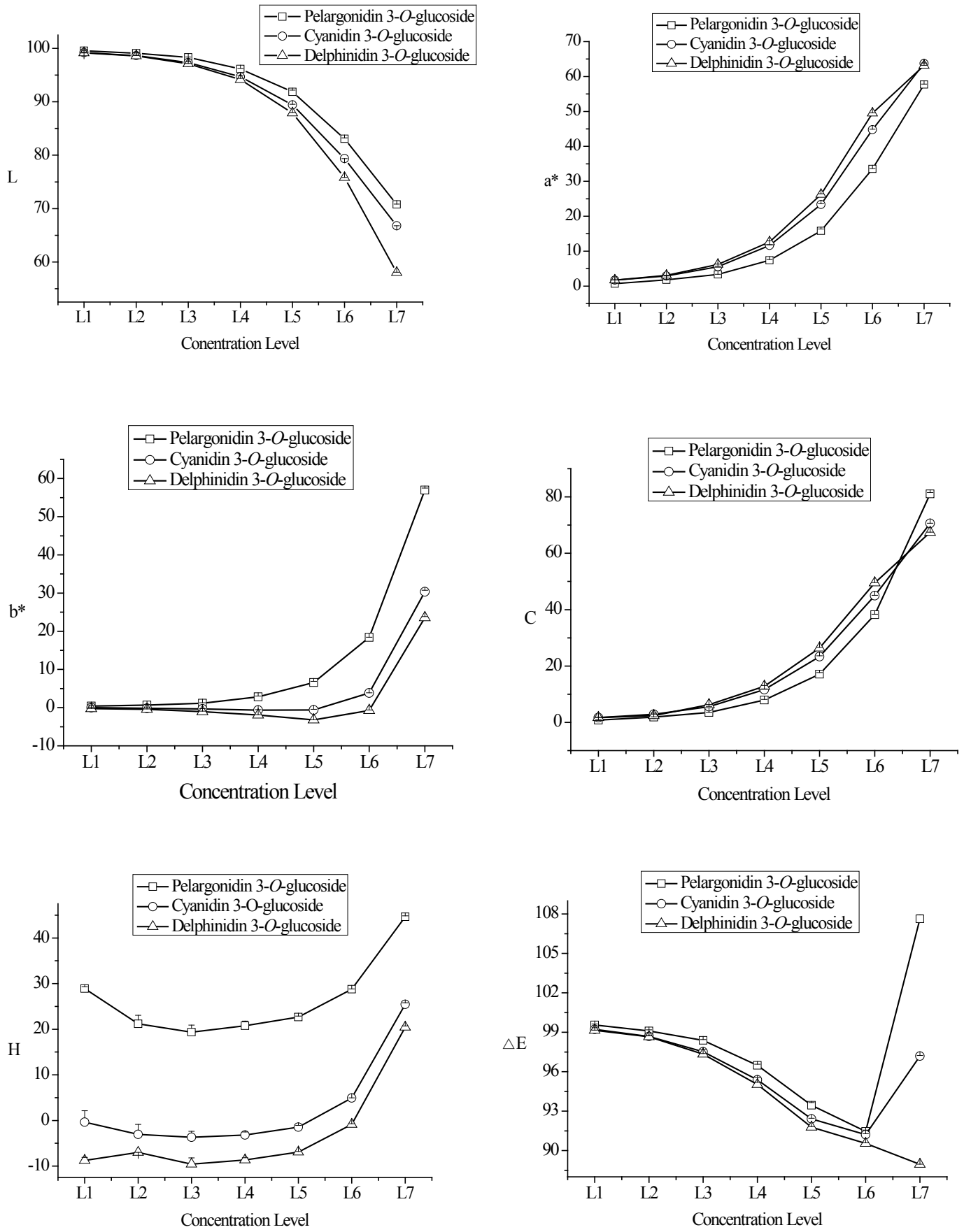

FIGURE 2

The colour of pelargonidin 3-O-glucoside, cyanidin 3- $O$-glucoside and delphinidin 3-O-glucoside. (L: lightness, $\mathrm{a}^{*}$ : red or green colour, $\mathrm{b}^{*}$ : yellow or blue colour, $\mathrm{C}$ : chroma, $\mathrm{H}$ : tone, $\triangle \mathrm{E}$ : colour difference).

Furthermore, the impact of hydroxyl substitution at the C3' position was greater on the colour than the substitution at C5'. The results of a previous study can partially explain this phenomenon (Pereira et al., 1997). The hydroxyl substitutions on the B-ring cause an increase in resonance through the $\mathrm{C} 2-\mathrm{C} 1$ ' bond. The hydroxylation in the B-ring leads to the delocalisation of $\pi$ electrons throughout the molecule, thereby lowering the HOMO-LUMO (highest occupied molecular orbital-lowest unoccupied molecular orbital) energy gap.

The effect of hydroxyl groups at the $\mathrm{C} 3$ ' position on self-association was different from the effect of that on the bathochromic shift or colour. The addition of hydroxyl at the C3' position weakened the self-association, whereas the subsequent (further) addition of hydroxyl at the C5' position enhanced the self-association. However, the addition of hydroxyls at the $\mathrm{C}^{\prime}$ ' and $\mathrm{C}^{\prime}$ ' positions resulted in a decrease in $\mathrm{L}$ and $\mathrm{b}^{*}$ values, but caused an increase in the value of $\mathrm{a}^{*}$. Thus, in comparison with pelargonidin 3-O-glucoside, cyanidin 3-O-glucoside produced a darker colour, with more intensity in the red and blue colours, and reduced yellow colour. 


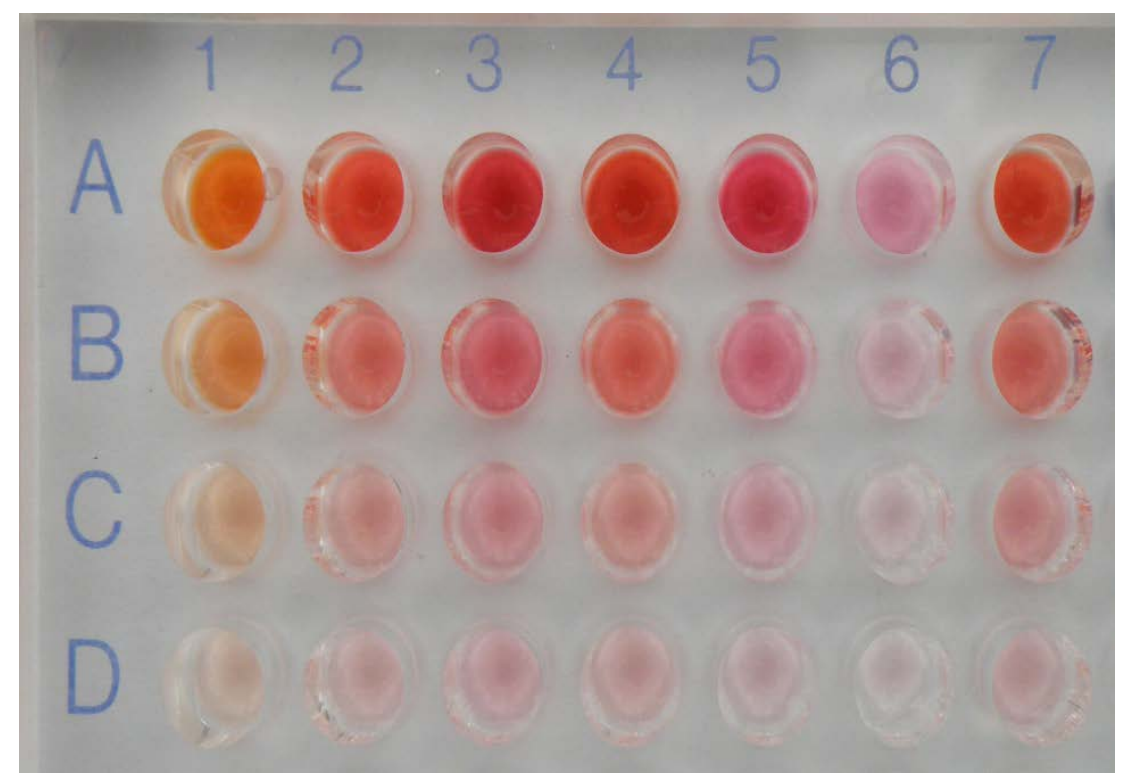

FIGURE 3

The colour of seven anthocyanins in an aqueous alcohol solution(1: pelargonidin 3-O-glucoside, 2: cyanidin 3-O-glucoside, 3: delphinidin 3-O-glucoside, 4: peonidin 3-O-glucoside, 5: malvidin 3- $O$-glucoside, 6: malvidin 3,5- $O$-diglucoside, 7: cyanidin 3-O-rutinoside; A, B, C and D correspond to concentrations L7, L6, L5 and L4 respectively.

\section{Effect of methoxy substitution on B-ring on colour}

Pelargonidin 3-O-glucoside $(\mathrm{H}, \mathrm{OH}, \mathrm{H})$, peonidin 3-O-glucoside $\left(\mathrm{OCH}_{3}, \mathrm{OH}, \mathrm{H}\right)$ and malvidin 3-O-glucoside $\left(\mathrm{OCH}_{3}, \mathrm{OH}, \mathrm{OCH}_{3}\right)$ differ in their pattern of $-\mathrm{OCH}_{3}$ substitutions on the B-ring (Fig. 1). The $\lambda_{\max }$ increased in the following order: pelargonidin 3-O-glucoside, peonidin 3-O-glucoside and malvidin 3-O-glucoside. The difference in $\lambda_{\text {max }}$ between pelargonidin 3-O-glucoside and peonidin 3-O-glucoside was bigger than that between peonidin 3-O-glucoside and malvidin 3-O-glucoside (Table 1). These results indicate that the bathochromic shift increased with increase in the number of methoxyl substitutions on the B-ring, and a methoxy group at the C3' position had a greater impact on the bathochromic shift than that at the $\mathrm{C} 5^{\prime}$ position. These results are similar to the results observed for the hydroxyl substitution on the B-ring (pelargonidin 3-O-glucoside, cyanidin 3-O-glucoside and delphinidin 3-O-glucoside).

Peonidin 3-O-glucoside and malvidin 3-O-glucoside had lower $\mathrm{L}, \mathrm{b}^{*}$ and $\mathrm{H}$ values and a higher $\mathrm{a}^{*}$ value than pelargonidin 3-O-glucoside (Fig. 4). The difference in $\mathrm{L}$ and $\mathrm{a}^{*}$ values between pelargonidin 3 - $O$-glucoside and peonidin 3-O-glucoside was larger than that between pelargonidin 3-O-glucoside and malvidin 3-O-glucoside. Peonidin 3-O-glucoside showed a slight blue hue at L2 to 4 (negative $\mathrm{b}^{*}$ and $\mathrm{H}$ value), while malvidin 3-O-glucoside displayed a higher extent of blue hue than that of peonidin 3-O-glucoside. The chromas of peonidin 3-O-glucoside across L1 to L6 were stronger than those of pelargonidin 3-O-glucoside, but weaker than those of pelargonidin 3-O-glucoside at the highest concentration. The value of chroma for malvidin 3-O-glucoside was less than that of peonidin 3-O-glucoside. As to their $\mathrm{H}$ value, pelargonidin 3-O-glucoside showed the strongest yellow hue, whereas malvidin 3-O-glucoside showed a blue hue. The differences in $\Delta \mathrm{E}$ values at lower concentrations were small, but became larger at higher concentrations. Thus, differences in their colour were observed visually (the difference of $\Delta \mathrm{E}$ value between them is higher than 1, Fig. 3).

These results indicate that methoxyl on the B-ring contributed to a darker colour ( $\mathrm{L}$ value decreased), higher red colour ( $\mathrm{a}^{*}$ value increased), lighter yellow colour and deeper blue colour ( $\mathrm{b}^{*}$ and $\mathrm{H}$ value decreased), purer and brighter chroma ( $\mathrm{C}$ value increased); a methoxy substitution at the C3' position on the B-ring contributed more colour to the anthocyanin than that at the $\mathrm{C}^{\prime}$ ' position. Similar results were observed with effect of methoxy substitutions on the B-ring on bathochromic shift, and also with the effect of hydroxyl substitutions on colour. The effect of a methoxy substitution on the B-ring on colour ( $\left.\mathrm{L}, \mathrm{a}^{*}, \mathrm{~b}^{*}\right)$ was identical to its effect on self-association.

\section{Effect of hydroxyl/methoxy substitution at $\mathbf{C 3}^{\prime}$ on colour} Pelargonidin 3-O-glucoside $(\mathrm{H}, \mathrm{OH}, \mathrm{H})$, cyanidin 3-O-glucoside $(\mathrm{OH}, \mathrm{OH}, \mathrm{H})$ and peonidin 3-O-glucoside $\left(\mathrm{OCH}_{3}, \mathrm{OH}, \mathrm{H}\right)$ differ in their substitutions at position $\mathrm{C}^{\prime}$ on the B-ring (Fig. 1). A comparison of their $\lambda_{\text {max }}$ 's (Table 1) shows that a methoxy substitution at position $\mathrm{C} 3$ ' causes a bigger bathochromic shift than a hydroxyl substitution at position $\mathrm{C} 3$ '.

In terms of the colour of these three anthocyanins (Fig. 5), it was found that methoxy substitution at position C3' of peonidin 3-O-glusoside reduced the lightness ( $\mathrm{L}$ value) to a greater extent than that caused by the hydroxyl substitution at position $\mathrm{C}^{\prime}$ ' of cyanidin 3-O-glucoside. Substitution with a hydroxyl group at position $\mathrm{C} 3$ ' lowered the yellow colour (lower $b^{*}$ and $\mathrm{H}$ values) at higher concentrations (L6 and L7); however, it enhanced the blue hue at lower concentrations (negative $\mathrm{b}^{*}$ and $\mathrm{H}$ values at $\mathrm{L} 1$ to $\mathrm{L} 5$ ) compared with the effect of a methoxy substitution at position C3'. The 

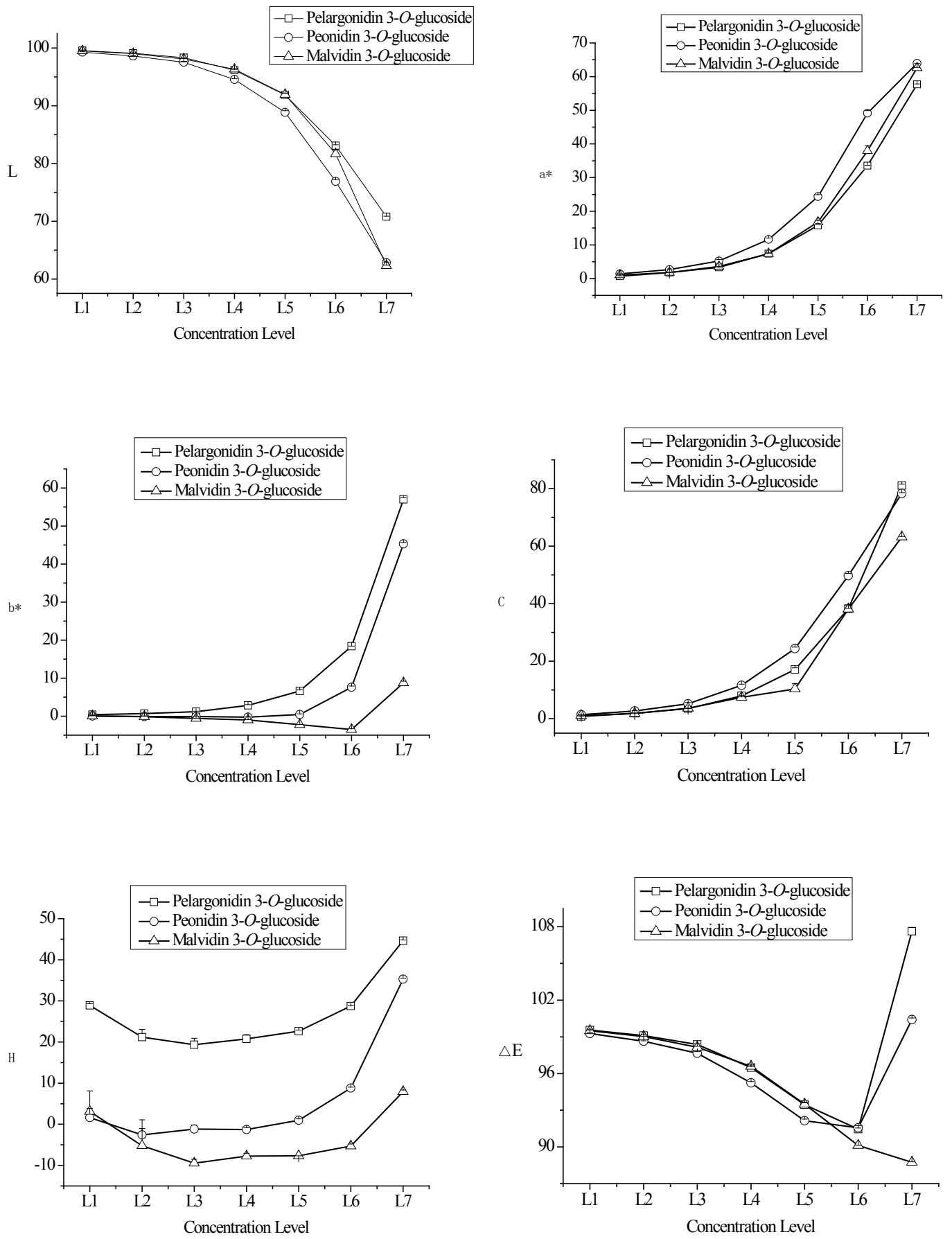

FIGURE 4

The colour of pelargonidin 3-O-glucoside, peonidin 3-O-glucoside and malvidin 3-O-glucoside. (L: lightness, $\mathrm{a}^{*}$ : red or green colour, $\mathrm{b}^{*}$ : yellow or blue colour, $\mathrm{C}$ : chroma, $\mathrm{H}$ : tone, $\Delta \mathrm{E}$ : colour difference).

hydroxyl and methoxy substitutions enhanced the red colour (increased $\mathrm{a}^{*}$ values). The methoxy substitution in peonidin 3-O-glucoside produced higher chroma values than that caused by hydroxyl substitution. Pelargonidin 3-O-glucoside presented the highest $\Delta \mathrm{E}$ value, and the differences in $\Delta \mathrm{E}$ value between peonidin 3-O-glucoside and cyanidin 3-O-glucoside were minor across L1 to L6 concentrations. The differences in the colour can be seen in Fig. 3.

It has been reported that peonidin 3-O-glucoside and cyanidin 3-O-glucoside (with two substituents on the B-ring) are located in the area of the orange hues in the $\left(\mathrm{a}^{*}, \mathrm{~b}^{*}\right)$ diagram, especially at $\mathrm{pH} 1.5$ (Heredia et al.,
1998). However, the results of the present study indicate that peonidin 3-O-glucoside and cyanidin 3-O-glucoside showed a red-purple colour at $\mathrm{pH} 3.50$. The published literature reports that the colour of malvidin 3-O-glucoside in red wines is consistent only with the uncharged blue quinonoidal base as a major coloured component of the equilibria at $\mathrm{pH} 3.6$ (Asenstorfer et al., 2003). These results were observed in the present study with the malvidin 3-O-glucoside solution at $\mathrm{pH}$ 3.5. A similar argument can be extended to the observation of purple hues in solutions of peonidin 3-O-glucoside and cyanidin 3-O-glucoside.

The hydroxyl or methoxy substitution at the $\mathrm{C} 3$ ' position 

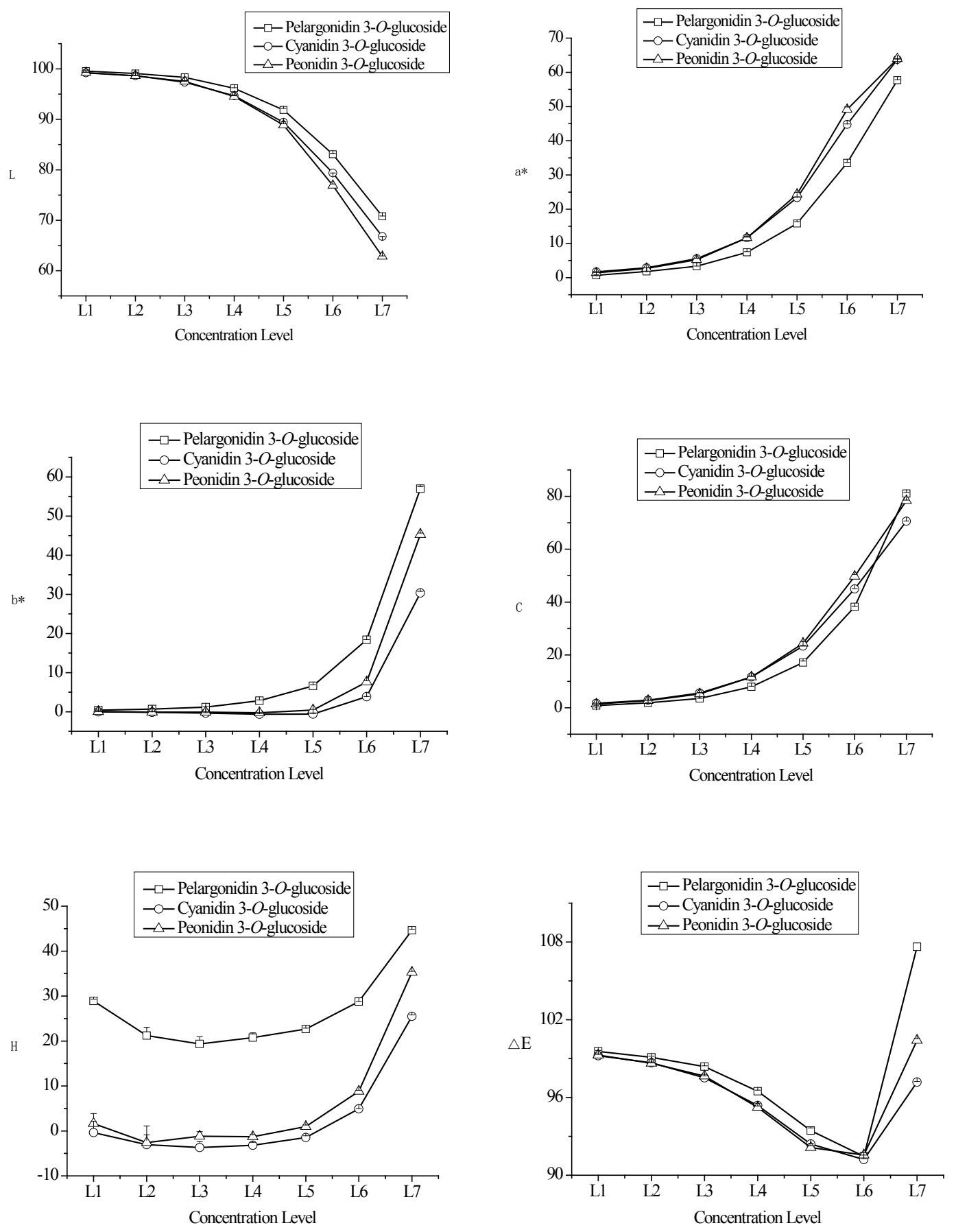

FIGURE 5

The colour of pelargonidin 3-O-glucoside, cyanidin 3-O-glucoside and peonidin 3-O-glucoside (L: lightness, $\mathrm{a}^{*}$ : red or green colour, $\mathrm{b}^{*}$ : yellow or blue colour, $\mathrm{C}$ : chroma, $\mathrm{H}$ : tone, $\Delta \mathrm{E}$ : colour difference).

on the B-ring enhanced the colour. However, the hydroxyl substitution at the $\mathrm{C} 3$ ' position on the B-ring was detrimental to self-association. The self-association of cyanidin 3-O-glucoside was responsible for 23 to $47 \%$ of the colour in its solution. Thus, it can be concluded that the addition of hydroxyl at the $\mathrm{C} 3$ ' position on the B-ring enhanced colour via intramolecular co-pigmentation instead of selfassociation.

Effect of glycoside substitution at $\mathrm{C} 3$ position on colour Cyanidin 3-O-glucoside and cyanidin 3-O-rutinoside differ in the presence of glucoside or rutinoside (a disaccharide) at the C3 position (Fig. 1). Substitution of rutinoside at the $\mathrm{C} 3$ position caused a larger bathochromic shift than substitution of glucoside at the same position (Table 1). Cyanidin 3-O-rutinoside was darker (lower $\mathrm{L}$ value) than cyanidin 3-O-glucoside (Fig. 6). It also showed a more intense red colour at L1 to L6 (higher a* value). However, the result was reversed at the $\mathrm{L} 7$ level. A stronger chroma (higher $\mathrm{C}$ value) across L2 to L7 and a weaker chroma at L1 of cyanidin 3-O-rutinoside were recorded. It showed intense yellow colour at L6 and L7 (higher $\mathrm{b}^{*}$ and $\mathrm{H}$ value), while it showed a weaker blue hue (negative $\mathrm{b}^{*}$ and $\mathrm{H}$ value) at lower concentrations ( $\mathrm{L} 1$ to $\mathrm{L} 5$ ). The difference in $\Delta \mathrm{E}$ value 
between them increased with an increase in concentration. The colour difference between them can also be observed in Fig. 3. These observations reveal that the substitution of rutinoside at the $\mathrm{C} 3$ position produced a stronger colour than the substitution of glucoside at the same position. The extent of self-association in cyanidin 3-O-rutinoside was less than that of cyanidin 3-O-glucoside. However, cyanidin 3-O-rutinoside presented the more enhanced colour. This probably can be attributed to a predominance of intramolecular co-pigmentation.
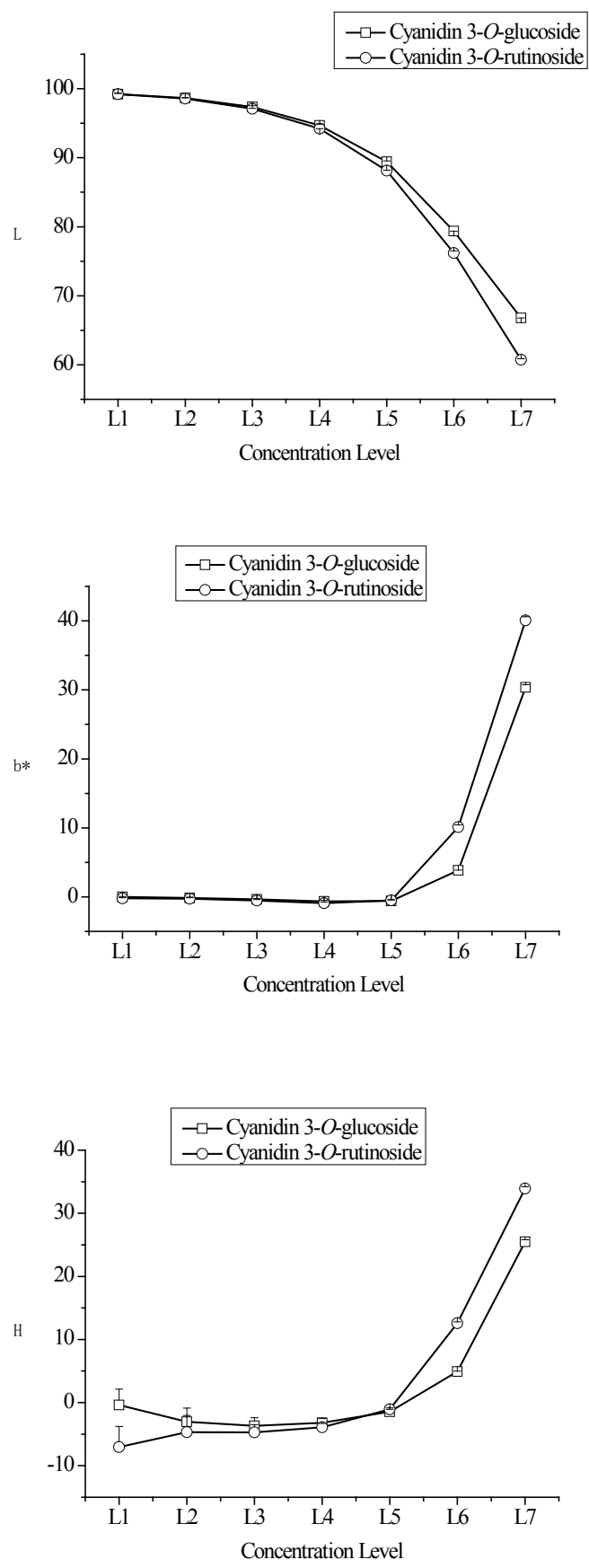

\section{Effect of glycoside substitution at the C5 position on colour}

Malvidin 3-O-glucoside and malvidin 3,5-O-diglucoside differ in their substitutions at the $\mathrm{C} 5$ position (Fig. 1). The presence of glucoside at the $\mathrm{C} 5$ position caused a stronger bathochromic shift, although the difference in $\lambda_{\max }$ between the two was small (2 nm, Table 1). As to their colour (Fig. 7), glucoside substitution at the C5 position resulted in lighter colour (higher L value), weaker red colour (lower a* value), strong blue colour (negative lower $\mathrm{b}^{*}$ and $\mathrm{H}$ value), and weaker chroma (lower $\mathrm{C}$ value). The difference in colour between them increased with increasing concentration
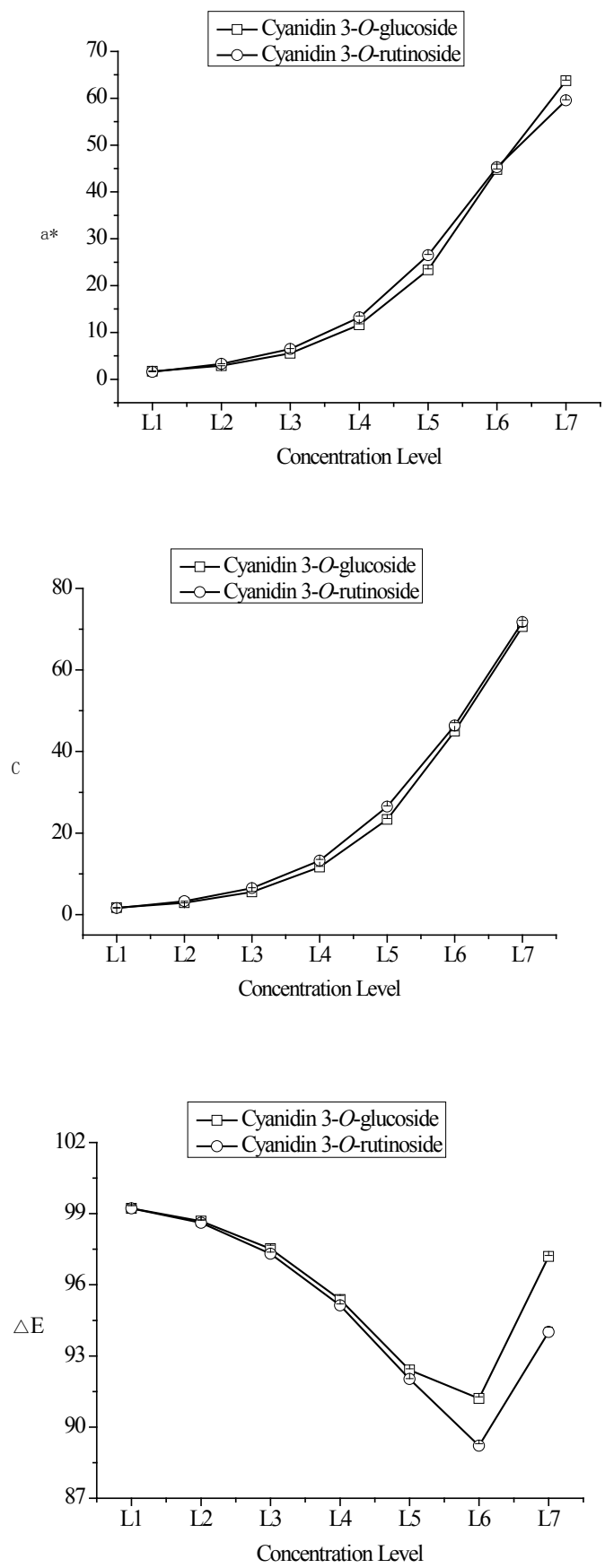

FIGURE 6

The colour of cyanidin 3-O-glucoside and cyanidin 3-O-rutinoside

(L: lightness, $\mathrm{a}^{*}$ : red or green colour, $\mathrm{b}^{*}$ : yellow or blue colour, $\mathrm{C}$ : chroma, $\mathrm{H}$ : tone, $\Delta \mathrm{E}$ : colour difference). 

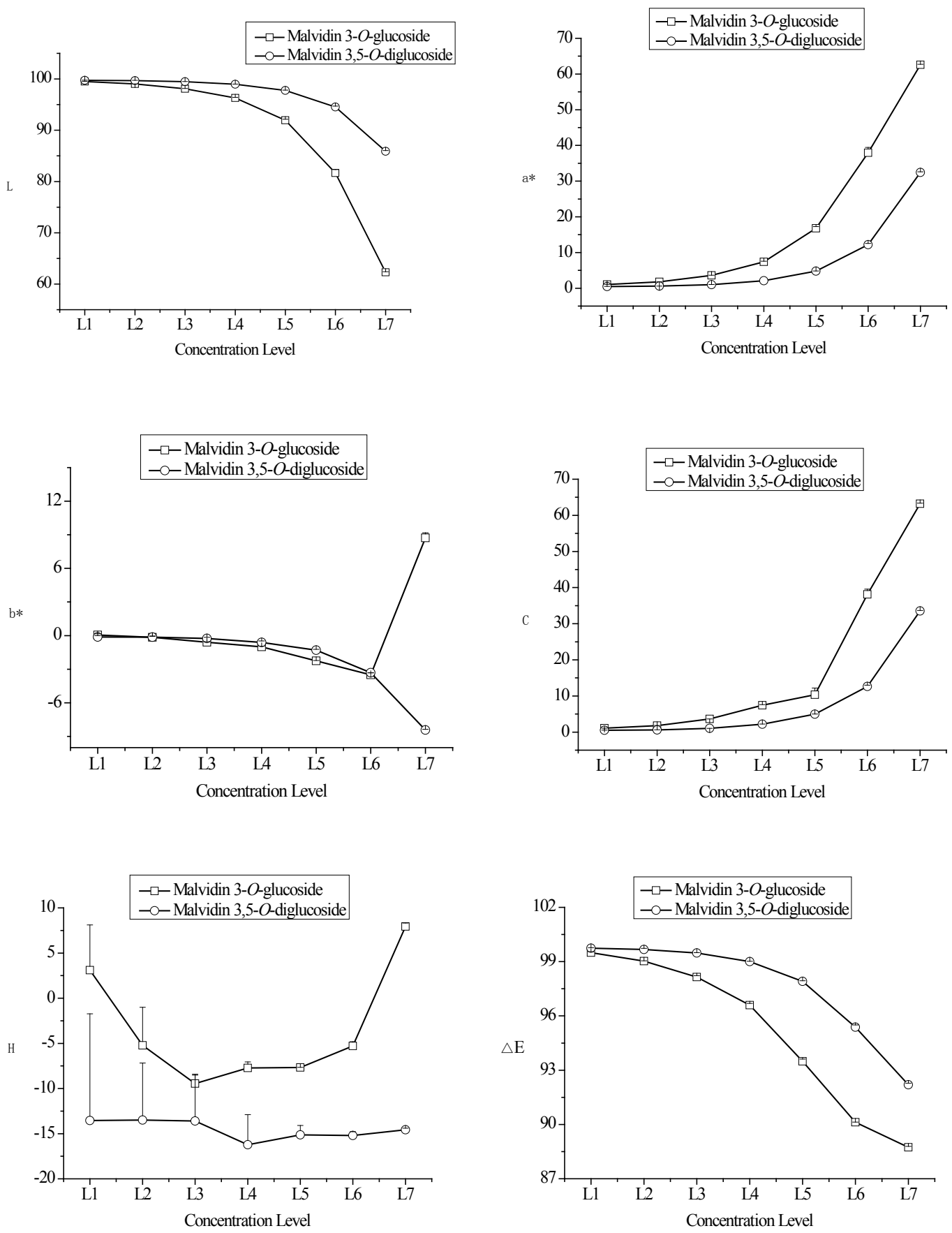

FIGURE 7

The colour of malvidin 3-O-glucoside and malvidin 3,5-O-diglucoside

(L: lightness, $\mathrm{a}^{*}$ : red or green colour, $\mathrm{b}^{*}$ : yellow or blue colour, C: chroma, $\mathrm{H}$ : tone, $\Delta \mathrm{E}$ : colour difference).

(Fig. 3). These results indicate that a glucoside substitution at the $\mathrm{C} 5$ position is detrimental to its colour. The extent of self-association in malvidin 3,5-O-diglucoside was also less compared with that of malvidin 3-O-glucoside, which can be a likely reason for the observed weakness in colour. A previous study reported that the glucose moiety at the $\mathrm{C} 5$ position (rather than that at the $\mathrm{C} 3$ position) played an important role in the self-association of these anthocyanidin 3,5-diglucosides (Hoshino et al., 1981a). However, Degenhardt et al. (2000) reported that malvidin 3-O-glucoside $(1.3 \mathrm{mg} / \mathrm{L})$ exhibited a lower visual detection threshold than malvidin 3,5-O-diglucoside $(4.7 \mathrm{mg} / \mathrm{L}$ ) (visual detection thresholds were established using a triangle test at
$\mathrm{pH}$ 3.6), which is in concurrence with the results obtained in this experiment. In addition, anthocyanin 3-monoglucosides showed greater resistance to nucleophilic attack compared with anthocyanin 3,5-diglucosides (Figueiredo et al., 1996).

These results indicate that self-association plays an import role in determining the colour of anthocyanins. Previous studies also reported that the colour of anthocyanins depended on their concentration (Asen et al., 1972; Ito et al., 2002). For anthocyanins, the major tautomeric form at $\mathrm{pH}$ values above 3 is the hemiacetal form, which is the reason why aqueous solutions of anthocyanins usually are colourless (Sweeny \& Iacobucci, 1983). On the other hand, the equilibria of anthocyanin tautomeric forms are also 
influenced by their concentration (Heredia et al., 1998). The literature reports that the planarity or non-planarity of the structure between the B-ring and C-ring of anthocyanins also has an effect on their colour properties (Sakata et al., 2006). Thus, substituent groups can have an effect on the colour via their ability to influence the equilibrium of tautomers, intramolecular co-pigmentation and self-association of anthocyanins, while self-association is closely related to the anthocyanin concentration.

\section{CONCLUSIONS}

Self-association plays an important role in anthocyanin colour. The self-association and colour of the seven anthocyanins investigated in this study were dependent on the structure and concentration of the anthocyanins. The anthocyanin colour was affected synthetically by the types and positions of the substituents in the anthocyanin. Cyanidin 3-O-glucoside and cyanidin 3-O-rutinoside improved colour more via intramolecular co-pigmentation than by selfassociation.

\section{LITERATURE CITED}

Alcalde-Eon, C., Escribano-Bailón, M.T., Santos-Buelga, C. \& RivasGonzalo, J.C., 2006. Changes in the detailed pigment composition of red wine during maturity and ageing: A comprehensive study. Anal. Chim. Acta $563,238-254$

Asen, S., Stewart, R.N. \& Norris, K.H., 1972. Co-pigmentation of anthocyanins in plant tissues and its effect on colour. Phytochem. 11, 11391144 .

Asenstorfer, R.E., Iland, P.G., Tate, M.E. \& Jones, G.P., 2003. Charge equilibria and pKa of malvidin-3-glucoside by electrophoresis. Anal Biochem. 318, 291-299.

Ayala, F., Echávarri, J.F. \& Negueruela, A.I., 1997. A new simplified method for measuring the colour of wines: I. Red and rosé wines. Am. J. Enol. Vitic. 48, 357-363.

Ayala, F., Echávarri, J.F. \& Negueruela, A.I., 1999. A new simplified method for measuring the colour of wines: III. All wines and brandies. Am. J. Enol. Vitic. 50, 359-363.

Boulton, R., 1996. A method for assessment of copigmentation in red wines. Paper presented at the 47th Annual Meeting of the American Society of Enology and Viticulture, Reno, NV, USA.

Boulton, R., 2001. The copigmentation of anthocyanins and its role in the colour of red wine: A critical review. Am. J. Enol. Vitic. 52, 67-87.

Cabrita, L., Fossen, T. \& Andersen, Ø.M., 2000. Colour and stability of the six common anthocyanidin 3-glucosides in aqueous solutions. Food Chem. 68, 101-107.

Castaneda-Ovando, A., De Lourdes Pacheco-Hernandez, M., PaezHernandez, M.E., Rodriguez, J.A. \& Galan-Vidal, C.A., 2009. Chemical studies of anthocyanins: A review. Food Chem. 113, 859-871.

Cavalcanti, R.N., Santos, D.T. \& Meireles, M.A.A., 2011. Non-thermal stabilization mechanisms of anthocyanins in model and food systems - An overview. Food Res. Int. 44, 499-509.

Cheminat, A. \& Brouillard, R., 1986. PMR investigation of 3-O-( $\beta$ d-glucosyl)malvidin structural transformations in aqueous solutions. Tetrahedron Lett. 27, 4457-4460.
Dangles, O., Saito, N. \& Brouillard, R., 1993. Anthocyanin intramolecular copigment effect. Phytochem. 34, 119-124.

Degenhardt, A., Hofmann, S., Knapp, H. \& Winterhalter, P., 2000. Preparative isolation of anthocyanins by high-speed countercurrent chromatography and application of the colour activity concept to red wine. J. Agr. Food Chem. 48, 5812-5818.

Dugo, P., Mondello, L., Errante, G., Zappia, G. \& Dugo, G., 2001. Identification of anthocyanins in berries by narrow-bore high-performance liquid chromatography with electrospray ionization detection. J. Agr. Food Chem. 49, 3987-3992.

Eiro, M.J. \& Heinonen, M., 2002. Anthocyanin colour behavior and stability during storage: Effect of intermolecular copigmentation. J. Agr. Food Chem. 50, 7461-7466.

Ellestad, G.A., 2006. Structure and chiroptical properties of supramolecular flower pigments. Chirality 18, 134-144.

Escribano-Bailón, M.T. \& Santos-Buelga, C., 2012. Anthocyanin copigmentation-evaluation, mechanisms and implications for the colour of red wines. Curr. Org. Chem. 16, 715-723.

Figueiredo, P., Elhabiri, M., Toki, K., Saito, N., Dangles, O. \& Brouillard, R., 1996. New aspects of anthocyanin complexation. Intramolecular copigmentation as a means for colour loss? Phytochem. 41, 301-308.

Gavara, R., Petrov, V., Quintas, A. \& Pina, F., 2013. Circular dichroism of anthocyanidin 3-glucoside self-aggregates. Phytochem. 88, 92-98.

Gonzáez-Manzano, S., Santos-Buelga, C., Dueñas, M., Rivas-Gonzalo, J.C. \& Escribano-Bailón, T., 2008. Colour implications of self-association processes of wine anthocyanins. Eur. Food Res. Technol. 226, 483-490.

Han, F.L., Jiang, Sh.M., He, J.J., Pan, Q.H., Duan, Ch.Q. \& Zhang, M.X., 2009. Anthocyanins in 'Cabernet Gernischet' (Vitis vinifera L. cv.) aged red wine and their colour in aqueous solution analyzed by partial least square regression. Food Sci, Biotechnol. 18, 724-731.

He, F., He, J.J., Pan, Q.H. \& Duan, C.Q., 2010. Mass-spectrometry evidence confirming the presence of pelargonidin-3-O-glucoside in the berry skins of Cabernet Sauvignon and Pinot Noir (Vitis vinifera L.). Aust. J. Grape Wine Res. 16, 464-468.

Heredia, F.J., Francia-Aricha, E.M., Rivas-Gonzalo, J.C., Vicario, I.M. \& Santos-Buelga, C., 1998. Chromatic characterization of anthocyanins from red grapes - I. pH effect. Food Chem. 63, 491-498.

Hoshino, T., Matsumoto, U. \& Goto, T., 1981a. Self-association of some anthocyanins in neutral aqueous solution. Phytochem. 20, 1971-1976.

Hoshino, T., Matsumoto, U., Harada, N. \& Goto, T., 1981b. Chiral exciton coupled stacking of anthocyanins: Interpretation of the origin of anomalous CD induced by anthocyanin association. Tetrahedron Lett. 22, 3621-3624.

Houbiers, C., Lima, J.C., Macüanita, A.L. \& Santos, H., 1998. Colour stabilization of malvidin 3-glucoside: Self-aggregation of the flavylium cation and copigmentation with the Z-chalcone form. J. Phys. Chem. B. $102,3578-3585$

Ito, F., Tanaka, N., Katsuki, A. \& Fujii, T., 2002. Why do flavylium salts show so various colours in solution? Effect of concentration and water on the flavylium's colour changes. J. Photoch. Photobio. A. 150, 153-157.

Lambert, S.G., Asenstorfer, R.E., Williamson, N.M., Iland, P.G. \& Jones, G.P., 2011. Copigmentation between malvidin-3-glucoside and some wine constituents and its importance to colour expression in red wine. Food Chem. 125, 106-115.

Li, Zh., Pan, Q.H., Cui, X.Y. \& Duan, Ch.Q., 2010. Optimization on anthocyanins extraction from wine grape skins using orthogonal test design. Food Sci. Biotechnol. 4, 1047-1053. 
Li, Zh., Pan, Q.H., Jin, Z.M., Mu, L. \& Duan, Ch.Q., 2011. Comparison on phenolic compounds in Vitis vinifera cv. Cabernet Sauvignon wines from five wine-growing regions in China. Food Chem. 125, 77-83.

Liang, Zh.C.H., Wu, B.H., Fan, P.G., Yang, Ch.X., Duan, W., Zheng, X.B., Liu, Ch.Y. \& Li, Sh.H., 2008. Anthocyanin composition and content in grape berry skin in Vitis germplasm. Food Chem. 111, 837-844.

Março, P.H. \& Scarminio, I.S., 2007. Q-mode curve resolution of UVvis spectra for structural transformation studies of anthocyanins in acidic solutions. Anal. Chim. Acta 583, 138-146.

Oliveira, J., Azevedo, J., Silva, A.M.S., Teixeira, N., Cruz, L., Mateus, N. \& De Freitas, V., 2010. Pyranoanthocyanin dimers: A new family of turquoise blue anthocyanin-derived pigments found in port wine. J. Agr. Food Chem. $58,5154-5159$.

Oliveira, J., Fernandes, V., Miranda, C., Santos-Buelga, C., Silva, A., De Freitas V. \& Mateus, N., 2006. Colour properties of four cyanidin-pyruvic acid adducts. J. Agr. Food Chem. 54, 6894-6903.
Pereira, G.K., Donate, P.M. \& Galembeck, S.E., 1997. Effects of substitution for hydroxyl in the B-ring of the flavylium cation. J. Mol. Struc.-Theochem. $392,169-179$.

Qin, Ch.G., Li, Y., Niu, W.N., Ding, Y., Zhang, R.J. \& Shang, X.Y., 2010. Analysis and characterisation of anthocyanins in mulberry fruit. Czech. J. Food Sci. 28, 117-126.

Sakata, K., Saito, N. \& Honda, T., 2006. Ab initio study of molecular structures and excited states in anthocyanidins. Tetrahedron 62, 3721-3731.

Sweeny, J.G. \& Iacobucci, G.A., 1983. Effect of substitution on the stability of 3-deoxyanthocyanidins in aqueous solutions. J. Agr. Food Chem. 31, 531-533.

Waterhouse, A.L. \& Kennedy, J.A., 2004. Red wine colour: Exploring the mysteries. American Chemical Society, Washington. 